\title{
Context Aware E-Support in E-Maintenance
}

\author{
Nikos Papathanassiou ${ }^{1,2}$, Christos Emmanouilidis ${ }^{2}$, \\ Petros Pistofidis ${ }^{1,2}$, and Dimitris Karampatzakis ${ }^{2}$ \\ ${ }^{1}$ Democritus University of Thrace, Greece \\ \{npapatha, pistofid\}@ceti.gr \\ ${ }^{2}$ ATHENA Research \& Innovation Centre, Greece \\ \{chrisem, dkara\}@ceti.gr
}

\begin{abstract}
Mobile learning is a powerful addition to the toolset offered by eLearning solutions. It can extend current e-Learning benefits to provide support and training everywhere, anytime and to anyone registered to have access and with affordable costs. Mobile devices with substantial computational, networking and storage capabilities are ubiquitous today. However, attempts to employ them in training are mostly addressing educational needs of end users with existing competencies in computing, such as students. We propose the development of a specially built mobile learning solution with the aim to provide e-Support to technicians within an e-Maintenance framework. This system will be backed and collaborate with a Learning Management System platform in order to provide on the spot aid and adequate supporting content to maintenance personnel.
\end{abstract}

Keywords: E-support, e-maintenance, mobile learning, e-training, context awareness.

\section{Introduction}

The reported research work aims to combine an integrated e-Maintenance framework with e-Training based on web technologies and supporting mobile devices. The e-Maintenance framework realizes platform-independent Condition-Based Maintenance by integrating an intelligent sensor network deployment, a Computerised Maintenance Management System (CMMS), a knowledge management system and an intelligent maintenance advisor, in the context of the WelCOM project [1]. It can provide an advanced, proactive type of Maintenance support that processes maintenance information throughout the plant, transparently providing it to anyone with authorized access. In this setting, our main aim is to study to what extend the e-Training system can be coupled with the other e-Maintenance components so as to provide context-aware support to shop-floor maintenance personnel and basic training on the system use to stakeholders.

E-Learning can be an effective way to train industrial employees, irrespective of their exact work placement due to some key attributes:

- First, it can offer time and location independent training, which has significant merit in workforce training. Industrial staff is hardly in position to attend formal classes and any probable pressure to become familiar with a new e-maintenance system could easily lead to overkill and negative attitude against the whole system. 
- Second, e-learning can provide a stimulating, intuitive and straight to the point way of delivering technical knowledge that can adapt and readily fit in the everyday schedule of a modern industrial plant.

- Third, e-learning content can be formatted and delivered directly to mobile devices as context-dependent technical support. This has the potential to accelerate the familiarity and facilitate the acceptance of the new training system by maintenance staff.

Modern mobile devices support connectivity, autonomy and offer computing and graphics capabilities that can be exploited to create appealing training environments at affordable cost. In the present work, supporting technical material is provided by a web server running a LMS (learning management system). The aim is to make it easy to locate directly the knowledge part that is requested on the mobile device and to update content regularly to reflect changes in maintenance practice. Our aim is to seek to adhere to e-learning standards, so as to increase the reusability of the content. Lately, our experimentation is focused on methods to seamlessly interconnect a customized mobile interface with the server side e-learning infrastructure. Based on this interconnection and on context-dependent data available from other WelCOM components, we will be able to provide contextualised support to the shop floor staff. Context may refer to different categories, such as user, environment, system, social and service [2]. In an industrial maintenance setting, the context can be quite wide. It may comprise information about specific machine, sensor readings, type of machinery operation, operation and maintenance history, type of maintenance action, possible failures, staff profile and role, as well as relevant services. This information should be communicated between e-Support and the rest of WelCOM e-Maintenance framework tools in a transparent and unobtrusive way, in order to simplify the delivered e-Support interface. Thus, e-Support is adequately offered via web services. Furthermore we seek to employ multiple modalities in human-computer interfaces, including speech interfaces.

\section{2 e-Learning and Mobile Learning}

In the last decade e-Learning has gained popularity and ubiquity, with relevant research carried out towards a wide spectrum of applications. Interconnectivity offered by ICT pushed the way for the implementation of solutions based on learnercentric Learning Theories, as well as to learning by direct interaction with others, typical in collaborative constructivism and groupware learning. Application of Constructivism and Activity Theory to mobile learning (m-Learning) in relation to acceptance by learners has been studied through questionnaires, highlighting significant advantages, such as learner initiative, improved interactivity and autonomy [3]. Attempts have also been reported to implement known learning theories to develop 3D multimedia courseware [4]. In research focused on engaging learning environments, Augmented Reality (AR) has been employed to deliver new platforms for easy creation of Virtual Scenes without previous programming knowledge [5].

Serious games are shown to bring significant benefits and personalisation options in order to create highly motivating and engaging learning experiences [6]. Employment of social networks in the Learning process has also been studied. 
Specifically, Web 2.0 elements, such as participant collaboration and virtual communities were evaluated, noting the connection between social learning networks and the "social constructivism" theory [7]. Work with elementary school pupils has shown how critical it is to deal with e-learning not as simply an extension of printed material but as medium with a multitude of advantages to exploit, like interactivity, multimedia and personalisation [8].

\subsection{E-Learning Standards}

The development costs for quality training material can be brought down by the adoption of Learning Standards that will promote content reusability, interoperability and discoverability. However, these standards still seem inefficient to support modern and engaging learner - system interactions such as multimedia and educational games. An important step to overturn this situation is a project named "Next Generation Learning Environment", carried out by ADL (Advanced Distributed Learning), founders of the SCORM model, one of the most widespread e-learning standards. The primary goal is to provide a standard that will support modern learning experiences, such as games, mobile learning, social networks, learner collaboration and mixed modalities. A straightforward model of integration in Learning Management Systems through improvements in the sequencing procedures has been presented [9].

\subsection{Industrial Training}

Currently demands for improved system productivity, availability, quality, safety and customer satisfaction, highlight the critical role of maintenance as an indispensable activity in industrial production. A significant number of complex factors must be taken into account in modern maintenance and fuel a demand for advanced ICT solutions [10]. The potential impact of an e-Maintenance framework on the implementation of a Condition Based Monitoring strategy is significant [11]. Mobile and collaborative technologies are enabling information and services to become available to the shop floor technical personnel, anywhere and anytime [12]. Furthermore, the concept of a Virtual Factory as an integrated virtual environment that facilitates knowledge and information sharing throughout all industrial production phases is offered as an open alternative to costly proprietary solutions for platform independent monitoring of production assets [13]. A learning engine capable of delivering learning material in the form of 3D knowledge objects to pilots and maintenance technicians in an aviation setting, emphasising access of maintenance technicians to "Learning on Demand (LoD)" instructions and trouble shooting references is an effective training enabler [14].

Industrial Maintenance can benefit from a common accreditation framework that will enhance workers mobility, support enterprise recruitment and enhance staff development. In Europe, such a framework has been set by EFNMS with the definition of competence requirements for maintenance managers and technicians [15], as well as by the PAS55 specification for optimising Asset Management practice, put forward by the Institute of Asset Management in the UK. 


\section{Problem Statement}

Developing e-Learning based e-Support for industrial maintenance technicians presents certain challenges:

- Mobile devices pose different restrictions due to monitor sizes, lack of keyboards and mice, limited battery life, unstable network connections and sometimes harsh working conditions. Despite the fact that there are devices with high-end characteristics and industrial design, restrictions have to be addressed to create a usable support application.

- Learners in a typical e-Learning setting would benefit by presenting the learning material gradually, in small sections, with intertwining comprehension questions so as to evaluate their performance and understanding as they proceed through the courses. Technicians on the shop floor would find all these as annoying distractions in their effort to access the exact information they need to perform their job, as they need precise, on the spot information.

- Procedures such as registration, login, course enrolment, searches through a breadth of learning content, are typical in every e-Learning setting. However, they would hinder the work of a technician, even more when he has to interact with other applications, as needed to complete the work orders.

Delivering technical support through mobile devices directly to the technicians on the shop floor, presents some unique benefits that we are planning to exploit:

- Real-time interactions with the supporting material could constitute an invaluable set of information regarding efficiency and appropriateness of the followed procedures and the relevant material. The underlying LMS would record all this information and technicians, engineers or technical managers would have access to it.

- Based on the above, a technician could select to attend a section of the e-learning that is relevant to a difficult part of his task. An engineer, supervisor or technical manager could pinpoint support elements that gather the most inquiries and initiate procedures to update and enrich them. As an example, the e-support system can notify the LMS that there are requests for maintenance actions on a specific machine. Based on this, the LMS can inform supervisors or managers and deliver a short web-based support relevant to the machine, so as to reach a better understanding of the problem at hand and possibly improve performance.

- Context definition in industrial e-support has not received sufficient attention and is usually restricted to localisation and specific user-context. Having a more thorough definition of context in this domain is among our research goals.

- Technically a major challenge we have to face up to is related to the fast and accurate delivery of e-support content to the mobile device. It is clear that under no circumstances this delivery should rely solely on wireless connections, due to number of obstacles, such as metal wall surfaces and roofs that are present in a factory. One solution that was examined initially was to transfer permanently all the supporting material inside the mobile devices. This idea is not satisfactory, first because the volume of this information could easily overload a mobile device and more importantly, it would negate the whole idea of intelligent interaction and collection of feedback by the LMS. Apart from breaking down the content into small 
entities, the Learning Objects, a potential solution to this problem is for the technician to be provided only with relevant for his job content. Avoiding redundancies on content delivery, all these objects will undergo custom compression procedures before their transfer to the mobile devices so as to minimize latency. Furthermore, a dynamic caching mechanism can be used to temporarily store large content entities, such as videos, to enhance the user experience.

- Intuitive environment and simplicity are two prerequisite qualities of the proposed solution, in order to minimize the case of rejection from low ICT-skilled users. Although the role of the supervisor is crucial and it is enhanced through the whole e-maintenance system, the e-support platform is expected to be gradually enriched at such level that it will allow for simple tasks to be performed without further human intervention and authorization. Thus, shop floor supervisors can focus on higher level activities.

- Another aspect of simplicity deals with the creation of new content. It is impractical to design a system that will need an ICT expert each time a new element, such as an image, video or technical document has to be added in the supporting database. Therefore, we opt for procedures that will be easily accessible to different staff categories inside an enterprise. The learning support software that will help the creation of new learning objects from raw support data is currently being developed.

A simple sequence diagram, showing the involved processes, invoked by technical staff, is shown in Fig. 1.

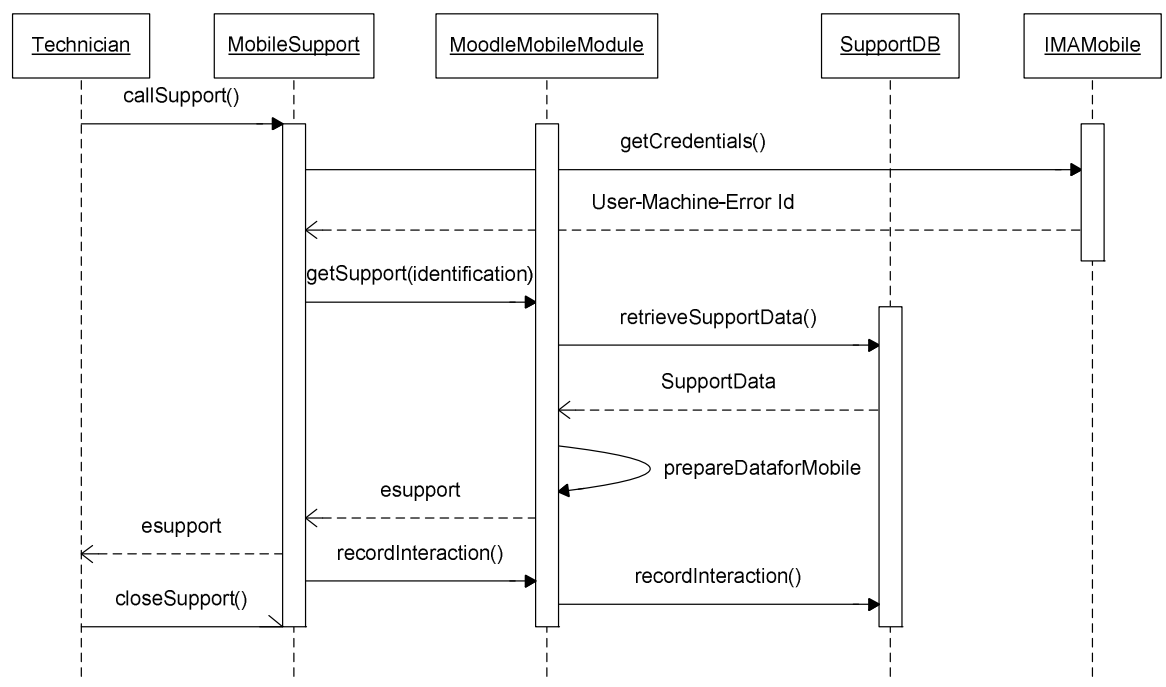

Fig. 1. E-Support Sequence Diagram

The above sequence diagram illustrates that the main e-support interaction commences when the technician on the shop floor requests support through the corresponding interface on the mobile device. The native application that is executed on the device proceeds to authorization - context definition procedures by requesting 
the relevant information from the other e-maintenance components (IMA) through web-services. Consequently, the mobile application contacts the corresponding Moodle module that gathers the requested support material from the learning database by searching based on meta-tags of Learning Objects. This material is processed, i.e. compressed by the module and is been transferred to the mobile device to be presented to the technician.

Essential in the procedure of delivering the appropriate content to each user is the formation of an extended learner profile that will contain constantly updated information about his knowledge, interactions and performance in theoretic learning and industrial work. This profile defines an important part of the context awareness and should be accessible at any time, so that it becomes possible to use the e-Learning system in order to enrich knowledge in the areas that he deems appropriate. It has to be also available to unit or line managers and maintenance supervisors, so they would have the necessary information to suggest improvements on the supporting material and possibly also on the maintenance policy implementation, with the aim to improve the technicians' performance.

Apart from the learner profile, all the learning and supporting content has to be broken down into fine-grained learning objects. These learning objects must be appropriately described and characterized by tags that will enable their search and their instant identification to be delivered to the field worker. The components of this eSupport system, within the WelCOM e-Maintenance architecture are shown in Fig 2.

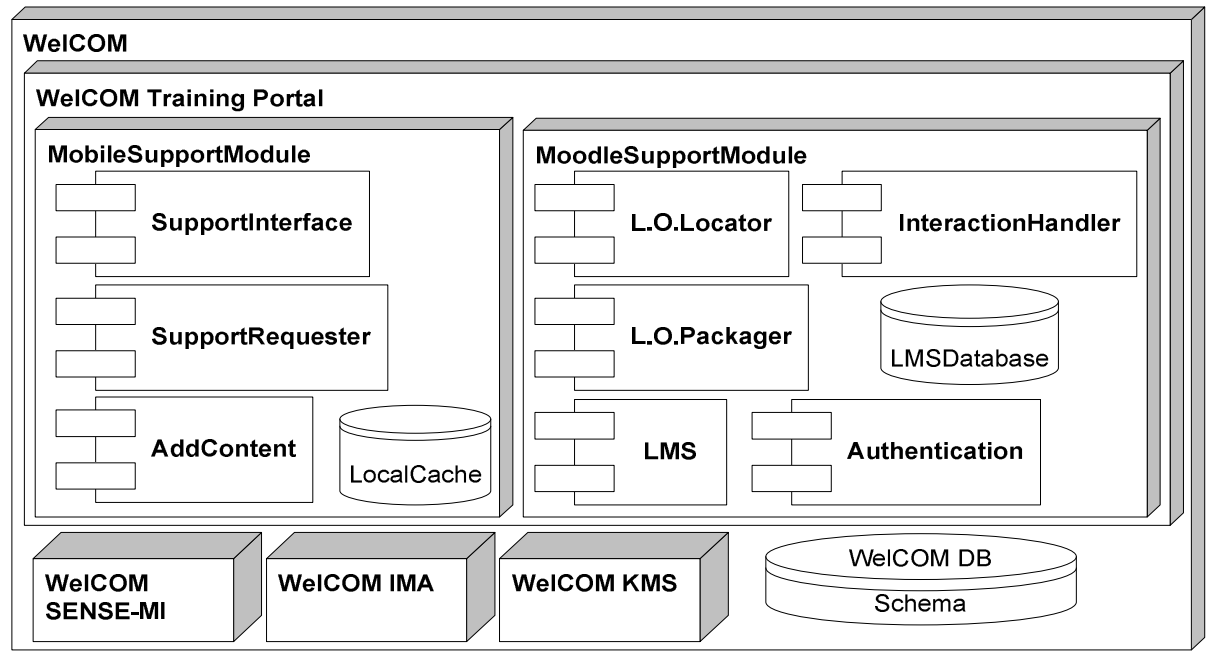

Fig. 2. WelCOM Training Portal Support Components

E-support data will be constantly updated and enhanced through the contribution of both managers and technicians. This purely practical knowledge content is expected to formulate a comprehensive library of information about machines and industrial procedures, organised through meta-tags and learning object interrelations in a way that is suitable to the end users, the technicians. As a step further, this library can be redeployed to other parts or subsidiaries of the industry or even to be traded as an asset with industries that use the same machinery. 


\section{Relevant Work}

e-Learning has been integrated in m-learning scenarios in various works. The combination of e-learning with m-learning has been tested in a school setting, in order to provide personalised learning [16]. Our work is differentiated in that (a) it addresses industrial training needs (b) knowledge is treated as Learning Objects that are dynamic so as to be updated with user-contributed content, e.g. by the technicians. Furthermore, the nature of the industrial processes themselves makes the provision of all assistive functions, including e-support, fundamentally important. Although a minor latency delay may be tolerated, the delivery of fast and contextually relevant support through mobile devices is one of the requirements in the WelCOM project. The placement of the e-support as a single module inside an integrated e-maintenance framework and the interconnectivity between the autonomous modules though webservices is also considered to be a key contribution.

\section{$5 \quad$ Further Work}

Currently, our focus is the examination in close collaboration with experts from the industry, of the latest SCORM and IMS standards and composition of a prototype model for our learning objects that will serve the requirements of our project. The development of the Moodle e-Support module has started based on the latest style and security guidelines.

\section{Conclusion}

Placing an e-Learning system as part of a working procedure in a demanding and dynamic setting, such as supporting industrial maintenance technicians, is a considerable challenge and the evaluation of its educational value is among our objectives. E-Support integration should be transparent, intuitive and offer streamlined access to contextually relevant content. It is considered that the integrated e-Training system, comprising both e-Learning and e-Support, would facilitate the adoption of a modern e-Maintenance system. Maintenance staff will be in position to employ more efficiently the offered technological solutions with the provision of context-dependent support and closely coupled e-Learning.

Acknowledgements. The author wish to acknowledge the enthusiasm and contribution by the WelCOM (GSRT grant 09SYN-71-856) project partner's staff, namely KLEEMANN Lifts, National Research Foundation, Prisma Electronics, GDT SA and ATLANTIS Engineering (welcom-project.ceti.gr).

\section{References}

1. Pistofidis, P., Emmanouilidis, C., Koulamas, C., Karampatzakis, D., Papathanassiou, N.: A Layered E-Maintenance Architecture Powered by Smart Wireless Monitoring Components. In: IEEE Conference on Industrial Technologies, ICIT 2012, Athens, Greece, May 19-21, pp. 390-395 (2011) 
2. Emmanouilidis, C., Koutsiamanis, R.-A., Tasidou, A.: Mobile Guides: Taxonomy of Architectures, Context Awareness, Technologies and Applications. Journal of Network and Computer Applications 36(1), 103-125 (2013)

3. Liaw, S.-S., Huang, H.-M.: Exploring Learners' Acceptance Toward Mobile Learning. In: Teo, T. (ed.) Technology Acceptance in Education, pp. 145-157. SensePublishers (2011)

4. Noordin, S., Ahmad, W.F.W.: Implementation of design and learning theories in multimedia courseware development: Lines \& Planes in 3-Dimensions. In: 2010 Int. Conf. on User Science and Engineering (i-USEr). IEEE (2010)

5. Jee, H.-K., et al.: An augmented reality-based authoring tool for E-learning applications. Multimedia Tools and Applications, 1-11 (2011)

6. Fradinho Oliveira, M., Andersen, B., Pereira, J., Seager, W., Ribeiro, C.: The Use of Integrative Framework to Support the Development of Competences. In: Ma, M., Fradinho Oliveira, M., Madeiras Pereira, J. (eds.) SGDA 2011. LNCS, vol. 6944, pp. 117-128. Springer, Heidelberg (2011)

7. Jones, K., et al.: Social Networks for Learning: Breaking Through the Walled Garden of the VLE. In: Abraham, A., Hassanien, A.-E. (eds.) Computational Social Networks, pp. 417-444. Springer, London (2012)

8. Huang, Y.-M., et al.: Empowering personalized learning with an interactive e-book learning system for elementary school students. Educational Technology Research and Development, 1-20 (2012)

9. Panar, A., Shumaker, T.M. (eds.): Sharable Content Object Reference Model (SCORM®) 2004 4th Edition Testing Requirements (TR) Version 1.1. Advanced Distributed Learning (ADL) Initiative (2009)

10. Arnaiz, A., et al.: Information and Communication Technologies Within E-maintenance. In: Holmberg, K., et al. (eds.), pp. 39-60. Springer, London (2010)

11. Jantunen, E., et al.: e-Maintenance: a means to high overall efficiency. In: Kiritsis, D., et al. (eds.) Engineering Asset Lifecycle Management, pp. 688-696. Springer, London (2010)

12. Emmanouilidis, C., Liyanage, J.P., Jantunen, E.: Mobile solutions for engineering asset and maintenance management. Journal of Quality in Maintenance Engineering 15, 92-105 (2009)

13. Sacco, M., Dal Maso, G., Milella, F., Pedrazzoli, P., Rovere, D., Terkaj, W.: Virtual Factory Manager. In: Shumaker, R. (ed.) Virtual and Mixed Reality, Part II, HCII 2011. LNCS, vol. 6774, pp. 397-406. Springer, Heidelberg (2011)

14. Christian, J., Krieger, H., Holzinger, A., Behringer, R.: Virtual and Mixed Reality Interfaces for e-Training: Examples of Applications in Light Aircraft Maintenance. In: Stephanidis, C. (ed.) HCI 2007. LNCS, vol. 4556, pp. 520-529. Springer, Heidelberg (2007)

15. Franlund, J.: Some European Initiatives in Requirements of Competence in Maintenance. In: Proc. of CM-MFPT 2008, 5th Int. Conf. on Condition Monitoring \& Machinery Failure Prevention Technologies, Edinburgh, UK (2008)

16. Nedungadi, P., Raman, R.: A new approach to personalization: integrating e-learning and m-learning. Educational Technology Research and Development, 1-20 (2012) 